\title{
REPRESENTAÇÃO BIDIMENSIONAL PARA UM MODELO NETWORK DEA
}

\author{
Bruno Guimarães Torres \\ Universidade Federal Fluminense \\ R. Passo da Pátria, 156 - São Domingos, Niterói - RJ \\ brunogtorres@hotmail.com \\ Juliana de Castro Reis \\ Universidade Federal Fluminense \\ R. Passo da Pátria, 156 - São Domingos, Niterói - RJ \\ juliana.dcreis@gmail.com \\ João Carlos Correia Baptista Soares de Mello \\ Universidade Federal Fluminense \\ R. Passo da Pátria, 156 - São Domingos, Niterói - RJ \\ jccbsmello@id.uff.br
}

\begin{abstract}
RESUMO
Esse artigo propõe uma representação bidimensional para a fronteira de eficiência para um modelo Network DEA (NDEA). A representação é baseada em um estudo anterior que desenvolveu uma generalização do método de representação bidimensional para o DEA clássico usando uma nova forma de linearização. Os valores dos inputs virtuais e dos outputs virtuais são utilizados para a elaboração do gráfico da fronteira de eficiência. A representação gráfica da fronteira de eficiência permite que gestores e tomadores de decisão não familiarizados com a programação linear e DEA entendam os resultados obtidos de maneira simples e clara. O gráfico obtido fornece informações sobre as eficiências da DMU e as metas que podem ser alcançadas por elas. Além disso, a representação obtida agrega informações tanto dos estágios internos, quanto do estágio global no mesmo gráfico para cada DMU.
\end{abstract}

Palavra-chave: Análise Envoltória de Dados; DEA; Eficiência; Representação bidimensional; Network DEA.

\begin{abstract}
This paper proposes a two-dimensional representation for the efficient frontier for a Network DEA (NDEA) model. The representation is based on a previous study that developed a generalization of the two-dimensional representation method for classical DEA using a new form of linearization. The values of virtual inputs and virtual outputs are used to draw the efficient frontier graph. The graphical representation of the efficiency frontier allows managers and decision makers unfamiliar with linear programming and DEA to understand the results obtained simply and clearly. The graph developed provides information about DMU efficiencies and the goals that can be achieved by them. In addition,
\end{abstract}


the representation obtained aggregates information from both internal and overall stages on the same graph for each DMU.

Keywords: Data Envelopment Analysis; DEA; Efficiency; Two-dimensional representation; Network DEA.

\section{Como Citar:}

TORRES, Bruno Guimarães; REIS, Juliana de Castro; SOARES DE MELLO, João Carlos. Representação Bidimensional para um Modelo Network DEA. In: SIMPÓSIO DE PESQUISA OPERACIONAL E LOGÍSTICA DA MARINHA, 19., 2019, Rio de Janeiro, RJ. Anais [...]. Rio de Janeiro: Centro de Análises de Sistemas Navais, 2019.

\section{INTRODUÇÃ̃O}

Devido à sua natureza matemática, os resultados obtidos pelos modelos DEA (Data Envelopment Analysis) e Network DEA são difíceis de interpretar para aqueles que não estão familiarizados com a programação linear. Por essa razão, as representações gráficas da fronteira de eficiência são muito úteis para facilitar o entendimento do DEA. Segundo Bana e Costa et al. (2016), essas representações são poderosas ferramentas de apoio para que os tomadores de decisão mostrem a posição de cada DMU em relação à fronteira eficiente ou busquem concentrações de DMUs em algumas áreas do gráfico.

A representação gráfica da fronteira de eficiência para o caso de um input e um output é o caso mais simples, onde o input é representado no eixo x e o output é representado no eixo y. Alguns pesquisadores têm trabalhado no fornecimento de técnicas alternativas para representar a fronteira DEA no plano na presença de mais variáveis (para uma revisão detalhada da literatura, ver Bana e Costa et al. (2016). No entanto, as técnicas propostas até o momento implicam em algumas dificuldades, como a visualização mais difícil com o aumento de variáveis, o uso de modelos DEA transformados e a falta de representação clara da fronteira de eficiência. Para evitar essas dificuldades, Bana e Costa et al. (2016) propuseram uma nova abordagem para a representação bidimensional da fronteira eficiente para modelos DEA clássicos que contorna as desvantagens das visualizações anteriores. Essa nova abordagem utiliza o input virtual e o output virtual para plotar as DMUs no gráfico e pode ser aplicada em modelos com vários inputs e vários outputs..

Para o Network DEA e outros modelos avançados de DEA, a representação gráfica da fronteira de eficiência é ainda mais restrita ou desconhecida, e não há muitos estudos sobre isso. Segundo Kao e Hwang (2014), a representação nesses modelos só é possível para os estágios internos da DMU, e não para o estágio global.

Torres et al. (2017) propuseram um modelo de representação bidimensional da fronteira de eficiência baseado no modelo de Bana e Costa et al. (2016) para o modelo Network DEA relacional multiplicativo proposto por Kao e Hwang (2008). No entanto, como o modelo de Kao e Hwang (2008) possui múltiplas soluções ótimas para os estágios internos da DMU, Despotis et al. (2016a) desenvolveram um modelo Network DEA em que os resultados das eficiências são únicos para casa estágio.

Assim, o presente estudo tem como objetivo propor um modelo de representação gráfica da fronteira de eficiência para o modelo Network DEA desenvolvido por Despotis et al. (2016a) com a agregação multiplicativa, generalizando o modelo proposto no artigo de Bana e Costa et al. (2016). Neste artigo, pressupõe-se que os inputs exógenos (entradas iniciais) que atuam no primeiro estágio do processo são transformados em variáveis intermediárias e utilizadas como inputs para o segundo estágio produzir os outputs exógenos (saídas finais).

Na próxima seção, apresentamos uma breve revisão do modelo de representação 
bidimensional proposto por Bana e Costa et al. (2016). Isto é seguido por uma revisão de literatura do modelo Network DEA a ser analisado na Seção 3. A Seção 4 introduz a representação gráfica bidimensional proposta para o Network DEA, e na Seção 5 um exemplo numérico é apresentado. Finalmente, a Seção 6 descreve as conclusões do artigo.

\section{REPRESENTAÇÃO BIDIMENSIONAL DA FRONTEIRA DE EFICÊNCIA}

Para possibilitar a representação bidimensional da fronteira de eficiência quando existem múltiplos inputs e múltiplos outputs, Bana e Costa et al. (2016) propuseram uma nova abordagem que utiliza o input virtual (a soma ponderada dos inputs) e o output virtual (a soma ponderada dos outputs) para plotar todas as DMUs no gráfico.

Para isso, foi necessário substituir a restrição de linearização clássica, uma vez que nos modelos DEA clássicos a restrição adicionada para linearizar os o modelo diz que, por exemplo, no modelo orientado a inputs, o input virtual é igual a 1. Assim, em um gráfico que compara o input virtual com o output virtual, todas as DMUs estariam localizadas na mesma linha vertical, o que não daria significado para a construção da representação. A nova restrição substitui a anteriormente citada e estabelece que a soma total dos pesos dos inputs é igual a 1, transformando o modelo CCR orientado a inputs no modelo (1).

$$
\begin{aligned}
& \sum_{\operatorname{Max}} \frac{\sum_{r=1}^{s} u_{r} y_{r o}}{\sum_{i=1}^{m} v_{i} x_{i o}} \\
& \text { s.t. } \\
& \sum_{i=1}^{m} v_{i}=1 \\
& \sum_{r=1}^{s} u_{r} y_{r j} \\
& \sum_{i=1}^{m} v_{i} x_{i j} \\
& u_{j}, v_{i} \geq 0, i=1, \ldots, m, r=1, \ldots, n
\end{aligned}
$$

Para evitar o uso dessa nova formulação, que é um problema de programação fracionária, os resultados obtidos no modelo CCR clássico podem ser utilizados para calcular o novo input virtual modificado e o novo output virtual modificado. Seja $S_{j}$ a soma total dos pesos dos inputs da DMU $j$ (2), onde $v_{i j}$ é o peso do input $i$ para DMU $j$. Calculamos $v^{\prime}{ }_{i j}$ como o peso modificado do input $i$ para DMU $j$ e $u_{r j}{ }_{r j}$, o peso modificado do output $r$ para a DMU $j$, usando (3) e (4).

$$
\begin{aligned}
& S_{j}=\sum_{i=1}^{m} v_{i j} \\
& v^{\prime}{ }_{i j}=\frac{v_{i j}}{S_{j}}
\end{aligned}
$$




$$
\text { SPOLN }
$$

Finalmente, o input virtual modificado $\left(I^{\prime}{ }_{j}\right)$ e o output virtual modificado $\left(\mathrm{O}^{\prime}{ }_{j}\right)$ para a DMU j são determinadas por (5) e (6), respectivamente.

$$
\begin{gathered}
I^{\prime}{ }_{j}=\sum_{i=1}^{m} v^{{ }^{\prime}}{ }_{i j} x_{i j} \\
O^{\prime}{ }_{j}=\sum_{r=1}^{s} u_{r j}{ }_{r j} y_{r j}
\end{gathered}
$$

Os valores de $I^{\prime}{ }_{j}$ e $O^{\prime}{ }_{j}$ são usados para representar qualquer DMU $j$ em um gráfico bidimensional. Nessa representação, a nova linearização transforma a fronteira de eficiência em uma linha reta desde a origem que corta o quadrante em $45^{\circ}$. Isso porque, para que uma DMU seja eficiente, seu input virtual modificado deve ser igual ao seu output virtual modificado.

Bana e Costa et al. (2016) estenderam seu modelo de representação bidimensional para modelos orientados a outputs e a casos de retornos variáveis de escala (modelo BCC). Além disso, provaram que a eficiência obtida pelo modelo modificado é a mesma obtida pelos modelos clássicos. Outro ponto importante é que a representação bidimensional proposta para as DMUs e sua distância da fronteira eficiente podem ser usadas para determinar o índice de eficiência geometricamente. Aplicações desta abordagem podem ser encontradas em Sow et al. (2016) e Reis et al. (2017).

\section{REVISÃO DO MODELO DE DESPOTIS ET AL. (2016)}

Despotis e Koronakos (2014) mostraram que, nos modelos Network DEA, as estimativas de eficiência obtidas pelo método de decomposição aditiva (CHEN et al., 2009) são enviesadas, ao favorecer indevidamente um estágio em detrimento do outro, enquanto aquelas obtidas pelo método multiplicativo (KAO E HWANG, 2008) não são únicas. Em seguida, os autores apresentaram uma nova abordagem para estimar índices de eficiência únicos e imparciais para os estágios individuais, que são então agregados para obter o índice de eficiência global da unidade avaliada. Essa nova abordagem foi chamada de "abordagem de composição", em oposição à abordagem de decomposição.

Em outro estudo, Despotis et al. (2016a) definiram a eficiência das duas etapas como em (7) e (8). Selecionando uma orientação a outputs para o primeiro estágio e uma orientação a inputs para o segundo estágio, onde os mesmos pesos intermediários são assumidos para ambos os estágios.

$$
E_{0}^{1}=\min \frac{\sum_{i=1}^{m} v_{i} x_{i 0}}{\sum_{d=1}^{D} w_{d} z_{d 0}}
$$

s.t. 


$$
\begin{aligned}
& \frac{\sum_{i=1}^{m} v_{i} x_{i j}}{\sum_{d=1}^{D} w_{d} z_{d j}} \geq 1, j=1,2, \ldots, n \\
& v_{i}, w_{d} \geq 0 ; i=1,2, \ldots, m ; d=1,2, \ldots, D
\end{aligned}
$$

$$
E_{0}^{2}=\max \frac{\sum_{r=1}^{s} u_{r} y_{r 0}}{\sum_{d=1}^{D} w_{d} z_{d 0}}
$$

s.t.

$\frac{\sum_{r=1}^{s} u_{r} y_{r j}}{\sum_{d=1}^{D} w_{d} z_{d j}} \leq 1, j=1,2, \ldots, n$

$u_{r}, w_{d} \geq 0 ; r=1,2, \ldots, s ; d=1,2, \ldots, D$

Anexando as restrições do modelo (7) ao modelo (8) e vice-versa, Despotis et al. (2016a) obtêm um modelo estendido, que possui a mesma solução ótima de seus respectivos modelos anteriores. Como esses novos modelos têm restrições comuns, podem ser considerados conjuntamente como um problema biobjetivo. Depois de aplicar a transformação $\mathrm{C}-\mathrm{C}$ (Charnes-Cooper), pode ser formulado e resolvido como um problema de programação linear multiobjetivo (MOLP), como em (9).

Em termos de MOLP, o vetor $\left(E_{0}^{1} \geq 1, E_{0}^{2} \leq 1\right)$ constitui o ponto ideal do problema biobjetivo (9) no espaço de funções objetivo. Como o ponto ideal geralmente não é atingível, resolver um MOLP significa encontrar o conjunto de soluções não dominadas na Fronteira de Pareto.

Existem diferentes abordagens para resolver um MOLP. Uma abordagem usual é a abordagem de escalarização, que transforma o MOLP em um problema de programação linear de objetivo único. Agregar aditivamente as funções objetivo e introduzir uma função de distância, são dois métodos alternativos para construir a função de escalarização. O ponto ótimo de Pareto derivado do modelo quando agregado aditivamente as funções objetivo pode ser não-exclusivo e, portanto, os índices de eficiência dos dois estágios também podem não ser exclusivos. Embora não seja muito provável que ocorra, esse é o caso em que um segmento da Fronteira de Pareto é paralelo à linha da função objetivo.

$$
\begin{aligned}
& E_{0}^{1}=\min \sum_{i=1}^{m} v_{i} x_{i 0} \\
& E_{0}^{2}=\max \sum_{r=1}^{s} u_{r} y_{r 0}
\end{aligned}
$$

s.t. 


$$
\begin{aligned}
& \sum_{d=1}^{D} w_{d} z_{d 0}=1 \\
& \sum_{d=1}^{D} w_{d} z_{d j}-\sum_{i=1}^{m} v_{i} x_{i j} \leq 0, j=1,2, \ldots, n \\
& \sum_{r=1}^{s} u_{r} y_{r j}-\sum_{d=1}^{D} w_{d} z_{d j} \leq 0, j=1,2, \ldots, n \\
& u_{r}, v_{i}, w_{d} \geq 0, i=1,2, \ldots, m, r=1,2, \ldots, s, d=1,2, \ldots, D
\end{aligned}
$$

Então Despotis et al. (2016a) empregaram a distância de Tchebycheff não ponderada ( $L_{\infty}$ norm) para localizar uma solução única na Fronteira de Pareto, minimizando o máximo dos desvios de $\left(\sum_{i=1}^{m} v_{i} x_{i 0}, \sum_{r=1}^{s} u_{r} y_{r 0}\right)$ do ponto ideal $\left(E_{0}^{1}, E_{0}^{2}\right)$. Isso é realizado pelo modelo minmax a seguir (10), onde $\delta$ denota o maior desvio.

$$
\begin{aligned}
& \min \delta \\
& \text { s.t. } \\
& \sum_{i=1}^{m} v_{i} x_{i 0}-\delta \leq E_{j o}^{1} \\
& \sum_{r=1}^{s} u_{r} y_{r 0}+\delta \geq E_{j o}^{2} \\
& \sum_{d=1}^{D} w_{d} z_{d 0}=1 \\
& \sum_{d=1}^{D} w_{d} z_{d j}-\sum_{i=1}^{m} v_{i} x_{i j} \leq 0, j=1,2, \ldots, n \\
& \sum_{r=1}^{s} u_{r} y_{r j}-\sum_{d=1}^{D} w_{d} z_{d j} \leq 0, j=1,2, \ldots, n \\
& u_{r}, v_{i}, w_{d}, \delta \geq 0, i=1,2, \ldots, m, r=1,2, \ldots, s, d=1,2, \ldots, D
\end{aligned}
$$

O modelo de resolução (10) significa procurar uma solução onde os desvios do ponto ideal são iguais e minimizados. Como mostrado na Figura 1, extraída de Despotis et al. (2016a), a solução minmax é D, que representa a interseção da Fronteira de Pareto (a linha $\mathrm{ABCD})$ e um raio do ponto ideal $\left(E_{0}^{1}, E_{0}^{2}\right)$ com declive (-1).

A principal vantagem do modelo (10) sobre o modelo que agrega aditivamente as funções objetivo e os modelos de decomposição (métodos multiplicativos e aditivos) é que fornece um ponto único, não necessariamente extremo (vértice), na Fronteira de Pareto, ou seja, valores exclusivos de eficiência para os dois estágios (DESPOTIS et al., 2016a).

Uma vez obtida uma solução ótima $\left(v^{i}, w^{i}, u^{i}\right)$ do modelo (10), os valores de eficiência de cada estágio para a unidade $j_{o}$ são como em (11) para o primeiro estágio e como em (12) para o segundo estágio. Pode-se notar que a eficiência do primeiro estágio é igual ao inverso do input virtual exógeno, e a eficiência do segundo estágio é igual ao output virtual exógeno. 


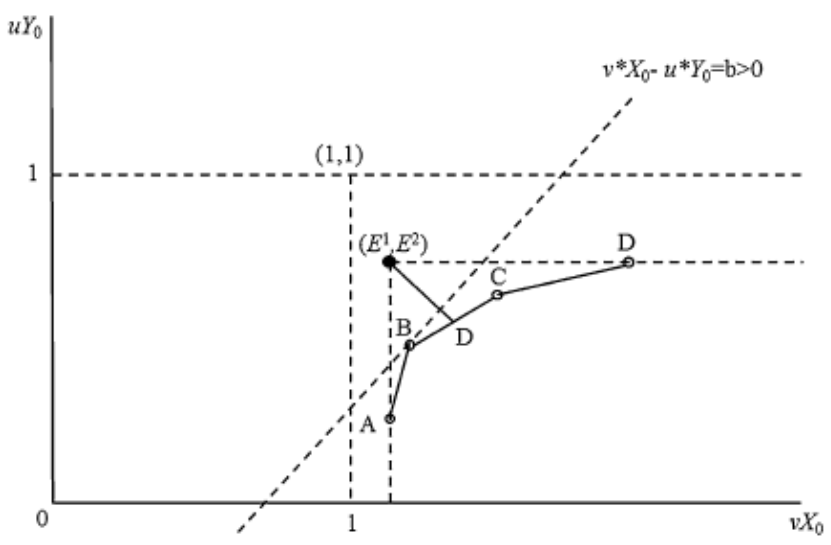

Figura 1 - A Fronteira de Pareto do MOLP (9) e a solução ótima do modelo (10) (Despotis et al., 2016a)

$$
\begin{gathered}
E_{0}^{1}=\frac{\sum_{d=1}^{D} w_{d} z_{d 0}}{\sum_{i=1}^{m} v_{i} x_{i 0}}=\frac{1}{\sum_{i=1}^{m} v_{i} x_{i 0}} \\
E_{0}^{2}=\frac{\sum_{r=1}^{s} u_{r} y_{r 0}}{\sum_{d=1}^{D} w_{d} z_{d 0}}=\sum_{r=1}^{s} u_{r} y_{r 0}
\end{gathered}
$$

Por fim, as eficiências dos dois estágios individuais precisam ser agregadas para obter a eficiência global. A eficiência global da unidade $j_{o}$ pode ser definida como a média aritmética das eficiências dos estágios individuais como em (13), ou podem ser agregadas multiplicativamente como em (14).

$$
\begin{aligned}
& E_{0}=\frac{1}{2}\left(E_{0}^{1}+E_{0}^{2}\right) \\
& E_{0}=E_{0}^{1} * E_{0}^{2}=\frac{1}{\sum_{i=1}^{m} v_{i} x_{i 0}} * \sum_{r=1}^{s} u_{r} y_{r 0}=\frac{\sum_{r=1}^{s} u_{r} y_{r 0}}{\sum_{i=1}^{m} v_{i} x_{i 0}}
\end{aligned}
$$

Posteriormente, Despotis et al. (2016b) desenvolveram uma abordagem geral de Network DEA para lidar com avaliações de eficiência em processos de múltiplos estágios. Essa abordagem também utiliza programação multiobjectivo como estrutura de modelagem e 
segue o mesmo paradigma de composição, em que as eficiências dos estágios são estimadas primeiro e a eficiência global do sistema é obtida posteriormente. .

\section{REPRESENTAÇÃO BIDIMENSIONAL PARA O MODELO DE DESPOTIS ET AL. (2016)}

A abordagem da composição foi desenvolvida por Despotis et al. (2016a), e leva em consideração que primeiro é necessário obter a eficiência dos estágios internos, e então agregá-los à eficiência do estágio global. Neste estudo, vamos nos concentrar na agregação multiplicativa.

$\mathrm{Na}$ agregação multiplicativa, a eficiência global pode ser obtida como a divisão do output virtual exógeno pelo input virtual exógeno. Portanto, a restrição de linearização não interfere no gráfico bidimensional e é possível representar a fronteira de eficiência global com o input virtual exógeno como o eixo $\mathrm{x}$ e o output virtual exógeno como o eixo $\mathrm{y}$. Quando o input virtual exógeno e o output virtual exógeno são iguais, a eficiência da DMU é 1. Assim, a fronteira de eficiência é definida como uma linha de 45 graus, como no modelo de Kao e Hwang (2008).

Além disso, como a eficiência do primeiro estágio é igual ao inverso do input virtual exógeno (como visto em 11), a eficiência dessa etapa é igual a 1 quando o input virtual exógeno é igual a 1. Portanto, pode-se notar que as DMUs eficientes no primeiro estágio são representadas no gráfico em uma linha vertical onde o input virtual exógeno é igual a 1.

Analogamente, a DMU somente é eficiente no segundo estágio se o output virtual exógeno for igual a 1 (como visto em 12). Assim, as DMUs eficientes no segundo estágio são representadas no gráfico em uma linha horizontal onde o output virtual exógeno é igual a 1 .

Em consequência, deve-se plotar as DMUs que são eficientes no primeiro e no segundo estágio no ponto $(1,1)$ do gráfico. Essas DMUs também são eficientes globalmente e são representadas na fronteira bidimensional, que é representada em uma linha de 45 graus, onde os valores dos dois eixos são os mesmos.

Outra característica dessa representação bidimensional é que as DMUs podem ser divididas em dois grupos. O primeiro grupo é composto pelas DMUs que são mais eficientes no primeiro estágio do que no segundo estágio, enquanto o segundo grupo é o oposto. Para determinar esses grupos, primeiro é necessário encontrar a fronteira onde a eficiência do primeiro estágio é igual à eficiência do segundo estágio. Como a eficiência da primeira fase é igual ao inverso do input virtual exógeno, e a eficiência da segunda etapa é igual ao output virtual exógeno, pode-se obter essa hipérbole, apresentada em (15).

$$
E_{0}^{1}=E_{0}^{2} \rightarrow \frac{1}{\sum_{i=1}^{m} v_{i} x_{i 0}}=\sum_{r=1}^{s} u_{r} y_{r 0} \rightarrow \frac{1}{I}=O
$$

As DMUs que são mais eficientes no primeiro que no segundo estágio são representadas abaixo da hipérbole, as DMUs que são mais eficientes no segundo estágio do que no primeiro estágio são representadas acima da hipérbole.

Pode-se observar que essa representação bidimensional, embora única para o estágio global, também contém informações sobre os estágios internos das DMUs. Diferentemente da representação proposta para o modelo multiplicativo relacional que 
apresenta uma representação de fronteira bidimensional para cada estágio e para o estágio global, separadamente (TORRES et al., 2016).

\section{EXEMPLO NUMÉRICO}

A abordagem desenvolvida nesse artigo foi aplicada no estudo de caso apresentado por Kao e Hwang (2008), que avaliou 24 seguradoras de Taiwan.

Primeiro, as eficiências de cada estágio foram obtidas, assim como os inputs virtuais exógenos e os outputs virtuais exógenos utilizados para representar a fronteira de eficiência bidimensional. Como este modelo fornece pesos únicos para as DMUs, pode-se usar os valores obtidos. Esses resultados estão descritos na Tabela 1.

A Figura 2 mostra a representação bidimensional para o modelo proposto. Observase que nenhuma DMU está representada na fronteira eficiente, como os resultados da Tabela 1 apontaram. A DMU mais eficiente é a DMU 12, que é a DMU mais próxima da fronteira de eficiência. Na representação bidimensional, observa-se que as DMUs eficientes no primeiro estágio são plotadas em $A ́ B$, onde o input virtual é igual a 1 , enquanto as DMUs eficientes no segundo estágio são plotadas em $B C A ́ B$, onde o output virtual é igual a 1.

Tabela 1 - Input virtual exógeno, output virtual exógeno e eficiências de cada estágio

\begin{tabular}{cccccc}
\hline DMU & $\begin{array}{c}\text { Input } \\
\text { Virtual }\end{array}$ & $\begin{array}{c}\text { Output } \\
\text { Virtual }\end{array}$ & $E_{0}^{1}$ & $E_{0}^{2}$ & $E_{0}^{1} * E_{0}^{2}=E_{0}$ \\
\hline 1 & 1.015435 & 0.7054 & 0.9848 & 0.7054 & 0.6947 \\
2 & 1.002908 & 0.6260 & 0.9971 & 0.6260 & 0.6242 \\
3 & 1.449275 & 1 & 0.6900 & 1 & 0.6900 \\
4 & 1.392564 & 0.4202 & 0.7181 & 0.4202 & 0.3018 \\
5 & 1.248284 & 0.9457 & 0.8011 & 0.9457 & 0.7577 \\
6 & 1.039609 & 0.4037 & 0.9619 & 0.4037 & 0.3883 \\
7 & 1.464772 & 0.4026 & 0.6827 & 0.4026 & 0.2748 \\
8 & 1.481921 & 0.4076 & 0.6748 & 0.4076 & 0.2750 \\
9 & 1.059659 & 0.2323 & 0.9437 & 0.2323 & 0.2192 \\
10 & 1.274697 & 0.5597 & 0.7845 & 0.5597 & 0.4391 \\
11 & 1.449485 & 0.2276 & 0.6899 & 0.2276 & 0.1570 \\
12 & 1 & 0.7596 & 1 & 0.7596 & 0.7596 \\
13 & 1.471887 & 0.3052 & 0.6794 & 0.3052 & 0.2073 \\
14 & 1.475579 & 0.4222 & 0.6777 & 0.4222 & 0.2861 \\
15 & 1.067122 & 0.6376 & 0.9371 & 0.6376 & 0.5976 \\
16 & 1.127269 & 0.3597 & 0.8871 & 0.3597 & 0.3191 \\
17 & 1.764291 & 0.6183 & 0.5668 & 0.6183 & 0.3504 \\
18 & 1.300221 & 0.3335 & 0.7691 & 0.3335 & 0.2565 \\
19 & 1.003814 & 0.4120 & 0.9962 & 0.4120 & 0.4104 \\
20 & 1.29668 & 0.6763 & 0.7712 & 0.6763 & 0.5216 \\
21 & 1.345171 & 0.2668 & 0.7434 & 0.2668 & 0.1984 \\
22 & 1.696353 & 1 & 0.5895 & 1 & 0.5895 \\
23 & 1.228350 & 0.5079 & 0.8141 & 0.5079 & 0.4135 \\
24 & 1.209629 & 0.1255 & 0.8267 & 0.1255 & 0.1037 \\
\hline 1 & & & & & \\
\hline
\end{tabular}

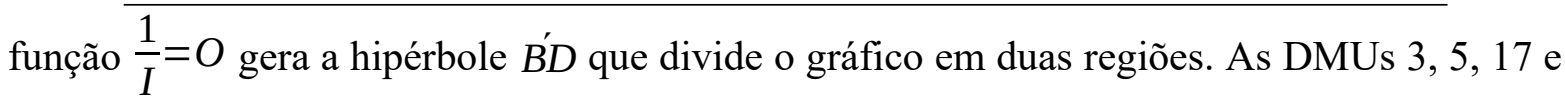


22, que estão representadas acima da hipérbole, são mais eficientes no segundo estágio do que no primeiro, enquanto as outras DMUs, representadas abaixo da hipérbole, são mais eficientes no primeiro estágio do que no segundo. Se uma DMU estivesse representada na hipérbole, teria a mesma eficiência em ambas as etapas, o que não aconteceu neste estudo.

Para cada DMU, pode-se obter a eficiência observando apenas o gráfico, porque a eficiência é definida como a divisão do output virtual pelo input virtual. Por exemplo, observando a Figura 2, pode-se estimar os valores de input virtual e output virtual para a DMU 12 , e assim obtêm-se a eficiência de $0,8 / 1=0,8$, que está próxima do valor efetivo de 0,7596. Um tomador de decisão que tenha acesso ao gráfico pode ter uma percepção geral da distribuição das eficiências das DMUs para o estágio global.

Também é possível obter uma estimativa do valor da eficiência de todas as DMUs para cada estágio apenas olhando para o gráfico. Por exemplo, para a DMU 12 o valor da eficiência do primeiro estágio é igual ao valor do inverso do valor do input virtual, que é igual a 1. E a eficiência do segundo estágio é igual ao valor do output virtual, que é próximo de 0,8 (valor real de 0,7596 ).

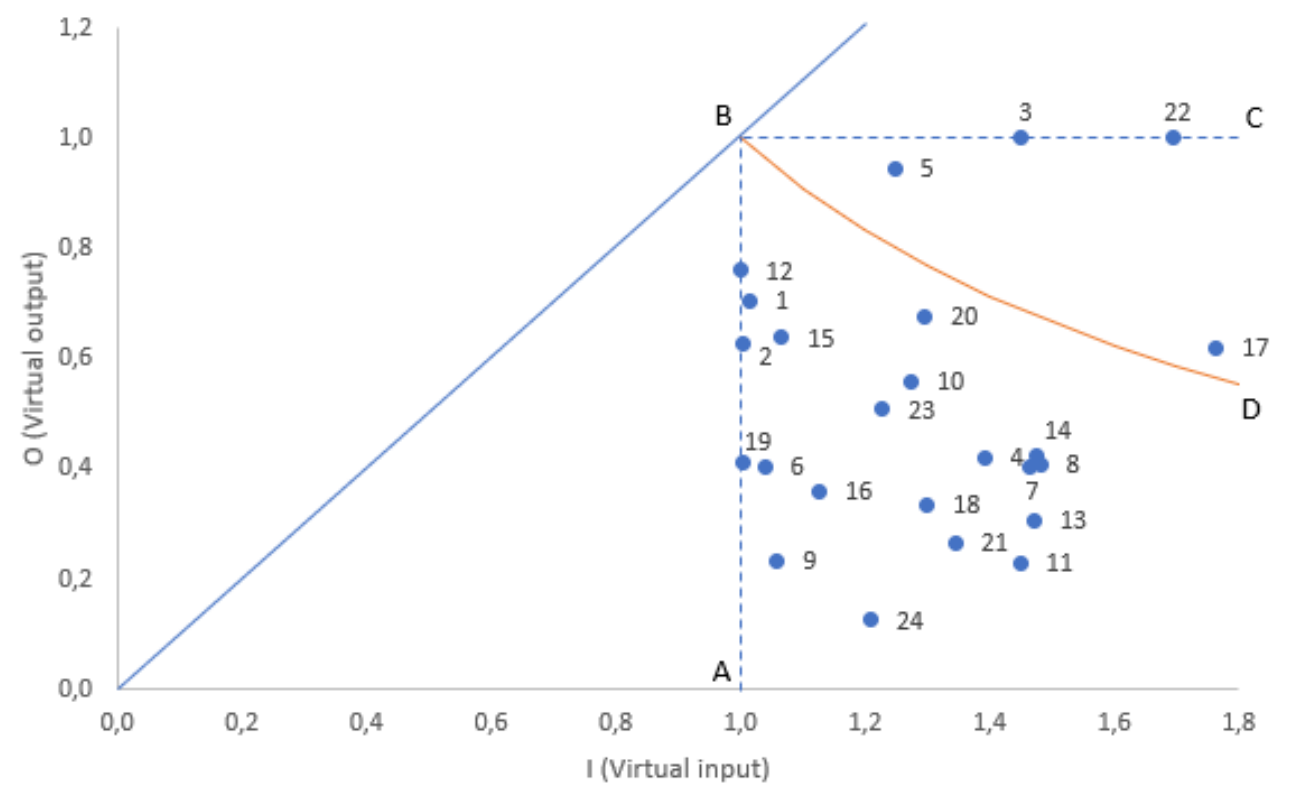

Figura 2 - Fronteira bidimensional para o modelo proposto

A representação proposta contém as mesmas propriedades que a proposta em Bana e Costa et al. (2016). No entanto, possui a vantagem de fornecer informações de mais de um estágio do Network DEA na mesma representação. Assim, um tomador de decisão com acesso à Figura 2 pode ter uma visão geral da eficiência global de cada DMU, bem como da eficiência de cada estágio interno da DMU.

\section{CONCLUSÕES}

Neste artigo, foi introduzida uma abordagem para fornecer uma representação bidimensional da fronteira eficiente para um modelo Network DEA desenvolvido por Despotis et al. (2016a), com a agregação multiplicativa.

Foi possível obter a representação bidimensional do modelo e a localização das DMUs. Foi proposto um gráfico para o estágio global apenas. No entanto, esse gráfico contém informações não apenas sobre o estágio global, mas também sobre o primeiro e o 
segundo estágios. Outra vantagem desta proposta é que não é preciso modificar os valores dos inputs virtuais e dos outputs virtuais para o desenvolvimento do gráfico.

A representação bidimensional pode ser uma ferramenta valiosa para ajudar os gerentes não familiarizados com a metodologia DEA a tomar decisões com base nos resultados encontrados nos modelos Network DEA, pois ela fornece informações quanto a proximidade de cada DMU da fronteira de eficiência de uma forma simples e visual, sem a necessidade de utilização de gráficos mais complexos.

Como sugestões para estudos futuros, propomos a representação da fronteira eficiente para as etapas internas do modelo de Despotis et al. (2016a). Além disso, propõe-se ampliar o modelo apresentado para outros modelos da Network DEA, como o modelo aditivo (CHEN et al., 2009), o modelo relacional (KAO, 2009) e os processos multi-estágio (DESPOTIS et al, 2016b).

\section{REFERÊNCIAS BIBLIOGRÁFICAS}

BANA E COSTA, C. A.; SOARES DE MELLO, J. C. C. B.; ANGULO MEZA, L. A new approach to the bi-dimensional representation of the DEA efficient frontier with multiple inputs and outputs. European Journal of Operational Research, v. 255, n. 1, p. 175-186, nov. 2016.

CHEN, Y. et al. Additive efficiency decomposition in two-stage DEA. European Journal of Operational Research, v. 196, n. 3, p. 1170-1176, 1 ago. 2009.

DESPOTIS, D. K.; KORONAKOS, G. Efficiency Assessment in Two-stage Processes: A Novel Network DEA Approach. Procedia Computer Science, 2nd International Conference on Information Technology and Quantitative Management, ITQM 2014. v. 31, p. 299-307, 1 jan. 2014.

DESPOTIS, D. K.; KORONAKOS, G.; SOTIROS, D. Composition versus decomposition in two-stage network DEA: a reverse approach. Journal of Productivity Analysis, v. 45, n. 1, p. 71-87, fev. 2016a.

DESPOTIS, D. K.; SOTIROS, D.; KORONAKOS, G. A network DEA approach for series multi-stage processes. Omega, v. 61, p. 35-48, jun. 2016 b.

KAO, C. Efficiency decomposition in network data envelopment analysis: A relational model. European Journal of Operational Research, v. 192, n. 3, p. 949-962, 2009.

KAO, C.; HWANG, S.-N. Efficiency decomposition in two-stage data envelopment analysis: An application to non-life insurance companies in Taiwan. European Journal of Operational Research, v. 185, n. 1, p. 418-429, 16 fev. 2008.

KAO, C.; HWANG, S.-N. Scale Efficiency Measurement in Two-Stage Production Systems. In: COOK, W. D.; ZHU, J. (Eds.). . Data Envelopment Analysis. International Series in Operations Research \& Management Science. [s.l.] Springer US, 2014. p. 119-135.

REIS, J. C. et al. Avaliação de eficiência das ferrovias brasileiras: Uma aplicação do método multicritério para seleção de variáveis em DEA e Representação Gráfica Bidimensional.

Revista ESPACIOS, v. 38, n. 14, 13 mar. 2017. 
SOW, O. et al. Efficiency Analysis of Public Transportation Subunits Using DEA and Bootstrap Approaches -- Dakar Dem Dikk Case Study. Journal of Mathematics Research, v. 8, n. 6, p. p114, 25 nov. 2016.

TORRES, B. G.; ALMEIDA, M. R.; SOARES DE MELLO, J. C. C. B. Representação Bidimensional do Network DEA: Um estudo de caso com avaliação de docentes. ANAIS DO XLIX SBPO. Anais... In: XLIX SBPO. Blumenau: SOBRAPO, 27 ago. 2017 